\title{
ENCEPHALOPATHIES
}

\section{ACUTE NECROTIZING ENCEPHALOPATHY}

The clinicopathological features of an acute necrotizing encephalopathy are described in a review of 13 consecutive children treated and 28 previously reported cases seen at various institutions in Japan. The onset was preceded by an upper respiratory infection, frequently treated with antipyretics and antibiotics. Aspirin was taken by only 2 patients. Symptoms of brain dysfunction, including impaired consciousness, convulsions, and vomiting, developed rapidly within 1 to 3 days. One third had hematemesis, one half had diarrhea, and all had liver enlargement without clinical jaundice. Hyperpyrexia, hyperventilation, and decorticate or decerebrate posturing occurred at the comatose stage. Twenty eight per cent died. In survivors, recovery of consciousness and neural function beginning after 6 to 10 days was slow, and serious sequelae such as spasticity, mental retardation, and seizures were common. Laboratory findings showed liver dysfunction, uremia, and hypoproteinemia. Liver histology was nonspecific and distinguished from Reye's syndrome. CSF protein was increased. CT and MRI revealed symmetric, multifocal areas of necrosis in the thalamus, white matter, brainstem, and cerebellum. The etiology of this previously unrecognized type of acute encephalopathy was not determined. (Mizuguchi $\mathrm{M}$ et al. Acute necrotizing encephalopathy of childhood: a new syndrome presenting with multifocal, symmetric brain lesions. I Neurol Neurosurg Psychiatry May 1995;58:555-561). (Respond: Dr M Mizuguchi, Department of Mental Retardation and Birth Defect Research, National Institute of Neuroscience, NCNP, 4-1-1 Ogawahigashi-cho. Kodaira, 187, Japan).

COMMENT. In addition to Reye's syndrome, the differential diagnosis included Wernicke's and Leigh's encephalopathies, carbon monoxide poisoning, acute disseminated encephalomyelitis, and acute hemorrhagic leukoencephalitis. The patients reported here appear to show some characteristics that differentiate this form of "acute toxic encephalopathy" from those already recognized. It is of interest that several patients $(16 \%)$ were retarded, and $16 \%$ had congenital anomalies, including ventricular septal defect, radial agenesis, and polydactyly. Evidence of recent viral infections (influenza A and B, coxackie A9, Rotavirus) was detected in 10 patients, and one patient had 3 paternal aunts who had died of Ekiri, a fulminant form of acute encephalopathy secondary to Shigella dysenteriae infection, prevalent in Japan up to the 1950 s.

\section{AMPHOTERICIN B-INDUCED ENCEPHALOPATHY}

Three children with refractory leukemia treated by bone marrow transplantation at the Children's National Medical Center, Washington, DC, developed encephalopathy, leukoencephalopathy, and parkinsonism after receiving high-dose amphotericin B for pulmonary aspergillosis. All 3 had previously been treated with high-dose chemotherapy and total body irradiation. MRIs showed basal ganglia, cerebellar, and cerebral atrophy, and frontal and temporal lobe white matter changes. One died and two recovered after withdrawal of the amphotericin, 1 having intellectual impairment. (Mott $\mathrm{SH}$, Packer RJ et al. Encephalopathy with Parkinsonian features in children following bone marrow transplantations and high-dose amphotericin B. Ann 